\title{
Optimization of Deep Rib High Speed Phase Modulators on 300mm Industrial Si-Photonics Platform
}

\author{
S.MONFRAY ${ }^{1}$, S.CREMER ${ }^{1}$, N.VUILLET ${ }^{1}$, E.DUBOIS ${ }^{2}$, F.DOMENGIE ${ }^{1}$, S.JAN ${ }^{1}$, F.BAILLE ${ }^{1}$, F.BEUF ${ }^{1}$ \\ ${ }^{1}$ STMicroelectronics, 850 Rue Jean Monnet, Crolles, 38920, France, \\ ${ }^{2}$ IEMN, Cité Scientifique, Avenue Poincaré, BP 60069, 59652 Villeneuve d'Ascq, France
}

stephane.monfray@st.com

\begin{abstract}
This paper highlights the optimization of Deep Rib High Speed Phase Modulators for 400G applications thanks to the optimal choice of structure and implants through a Design of Experiment analysis, including the proposal of a new Deep Rib HSPM with Z-implants. Results show 1,6dB gain in OMA (Optical Modulation of Amplitude) at the same cutoff frequency $\left(f \mathrm{c}=\left[2 \mathrm{Pi}^{*} \mathrm{RC}\right]^{-1}\right)$ compared to the previous generation [1] with optimized vertical implants, and up to $2,15 \mathrm{~dB}$ gain vs [1] with the new Z-implant Deep Rib device.
\end{abstract}

Keywords—photonics, Deep Rib PN junction modulators

\section{INTRODUCTION}

Silicon photonics technology provides unreached data densities and integration level while taking benefits from low cost and industrial manufacturing infrastructure of CMOS electronics [1]. In this paper, O-band Silicon PN junction modulators have been designed and integrated. In order to improve light confinement in the core for maximizing the modal overlap with PN junction, we optimized a Deep Rib architecture. The purpose of this paper is to demonstrate the optimization of the modulator to obtain the best optical performances and speed with optimized implants and structure thanks to a DOE (Design Of Experiments) analysis, and to propose a new combination of well implants (named Z-implants) with the optimized Deep Rib geometry to reach OMA (Optical Modulation Amplitude) value as high as $-2 \mathrm{dBm}$ with high cut-off frequency.

\section{DOE DEVICES INTEGRATION ON 300MM PHOTONICS PLATFORM}

A Design Of Experiments (DOE) for Deep Rib modulators was tested on our $300 \mathrm{~mm}$ Si-Photonics platform, including $\mathrm{Ge}$ Photodiodes, Si \& SiN waveguides and transitions (see Fig.1) for CWDM circuits.

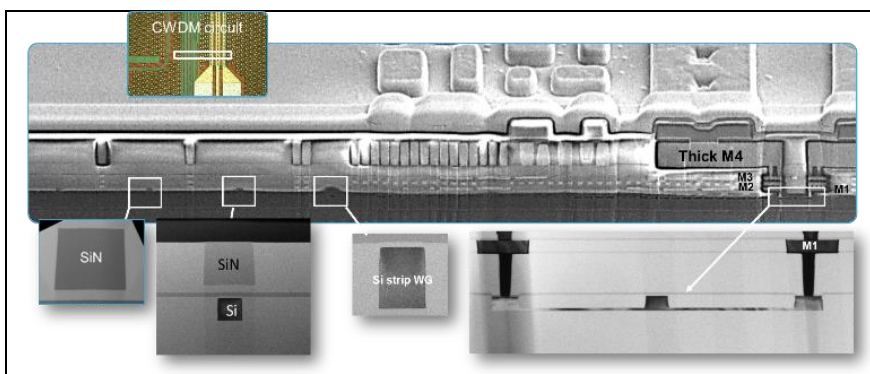

Fig.1: 300mm Si Photonic Platform including Si \& SiN waveguides, adiabatic transition, Thick M4 $\mathrm{Cu}$ level and Deep Rib HSPM modulators. Integrated Ge PD is not shown on the picture.

The Design of Experiments (DOE) for Deep Rib modulators was first established with variations on the junction position $\mathrm{Xj}$ (from $20 \mathrm{~nm}$ to $160 \mathrm{~nm}$ ), on the width of the modulator (W from $320 \mathrm{~nm}$ to $600 \mathrm{~nm}$ ) and on the distance d of access arms implants to the guide (from $100 \mathrm{~nm}$ to $400 \mathrm{~nm}$ ) with different levels of doping (Process of reference Arm Doping $x 1$ and highly doped Arm Doping $\mathrm{x} 20$ ), see figures $2 \mathrm{a}$ and $2 \mathrm{~b}$.

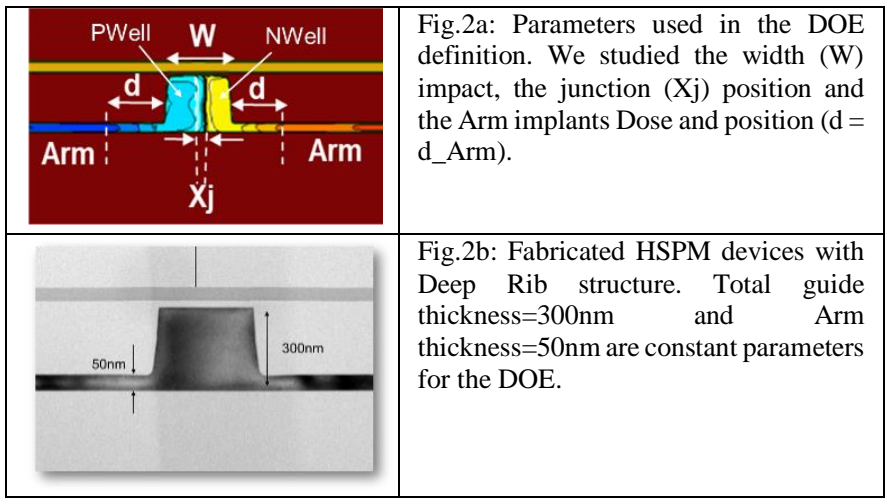

To extract the intrinsic cut-off frequency (fc) of the modulator PN junction, a so-called OPEN deembedding procedure has been applied to eliminate RC parasitics associated to the probing pads and interconnect layers from the topmost Metal4 to the inner Metal2 layers. This involves two distinct Sparameters measurements of the active device and of the passive OPEN structure, respectively. By subtracting the admittance of the OPEN from that of the modulator PN junction, the intrinsic $\mathrm{RC}$ product is subsequently easily determined to yield $\mathrm{f}_{\mathrm{c}}$. All RF measurements were performed with a reverse $\mathrm{PN}$ junction bias of $1.8 \mathrm{~V}$. The figure 3 shows that by reducing the distance to the waveguide of the Arm implants combined with a high dose (x20 vs industrial reference [1], $\mathrm{R}$ can be largely reduced leading to high fc.

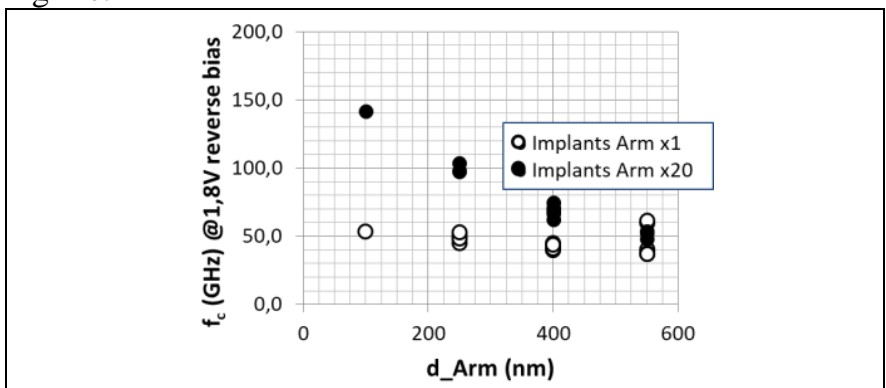

Fig 3 shows the measured cutoff frequency in function of d distance, for two levels of Arm Doping (x1 and x20) vs previous industrial reference [1])

However, this optimization has to take into account the impact on optical performances (in particular losses) induced by the 
doping modification. To explore this, we decided to monitor the OMA (Optical Modulation Amplitude) value for all structures of the DOE. OMA is defined as the difference between the optical power levels (Phigh and Plow) of the signal. The value of OMA is calculated based on the extracted HSPM parameters: $\alpha=$ total device Losses $(\mathrm{dB} / \mathrm{mm}), \mathrm{PS}=$ device Phase Shift $(\% / \mathrm{mm})$ and $\mathrm{L}=$ device length $(\mathrm{mm})$.

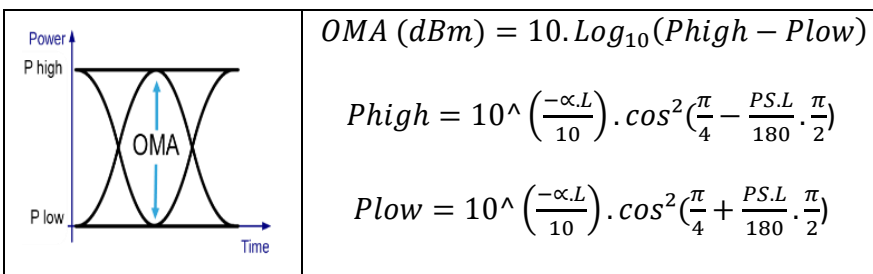

We highlighted that by increasing by a factor $20\left(\mathrm{~N}=1 \mathrm{e} 19 \mathrm{~cm}^{-3}\right)$ the implant dose in the access arms down a distance to the waveguide $d=250 \mathrm{~nm}$, the cutoff frequency could be highly improved (fig.3) without penalty on optical performances (fig. 4). For shorter distance, higher losses lead to OMA decrease.

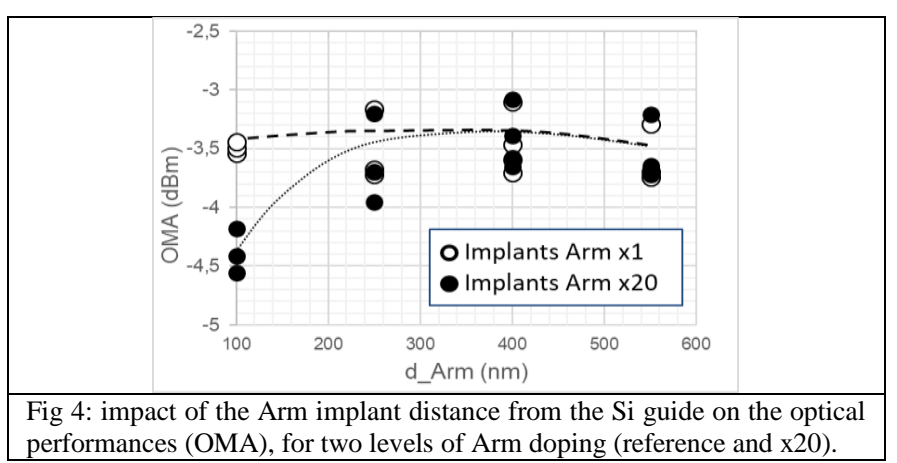

\section{OPTIMIZED DEVICES INTEGRATION WITH VERTICAL PN IMPLANTS}

In order to determine the best structure, we first performed TCAD simulations of all DOE structures to evaluate the access resistance, while other parameters (capacitances, losses, phaseshift) where measured on the fabricated devices.

Thanks to the DOE analysis, we determined that the best optical performances was obtained for $\mathrm{Xj}=60 \mathrm{~nm}$ and a width of $400 \mathrm{~nm}$. Experimentally, the closest measured device had a width of $370 \mathrm{~nm}$ and was highlighted as the best point of our OMA vs fc (=1/2PiRC) figure of merit (fig.5).

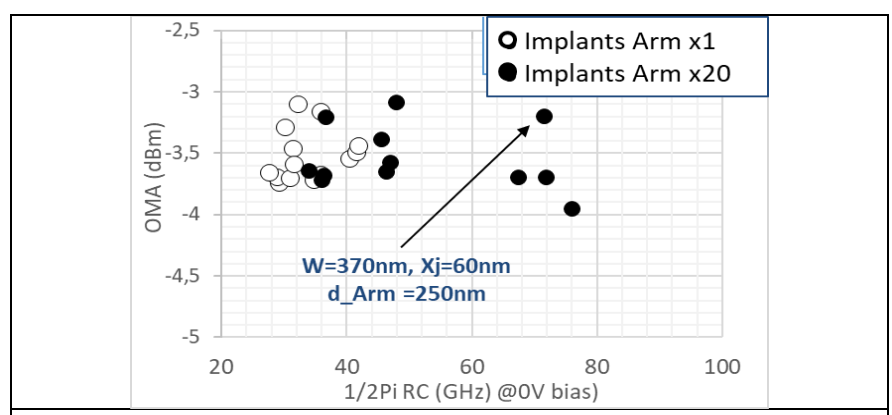

Fig 5: DOE results for different geometrical and implants parameters. Best structure for compromise OMA vs cut-off frequency is identified. $\mathrm{L}=2,4 \mathrm{~mm}$.
The same DOE was then measured with the RF structures (fig. 6) to extract the exact fc with a reverse bias of $1,8 \mathrm{~V}$, and confirmed the best performances for our identified experimental geometry $\quad\left(\mathrm{W}=370 \mathrm{~nm}, \quad \mathrm{Xj}=60 \mathrm{~nm}, \quad \mathrm{~d} \_\right.$Arm implant distance $=250 \mathrm{~nm}$ ) with the highest Arm implant dose (x20 vs the previous platform).

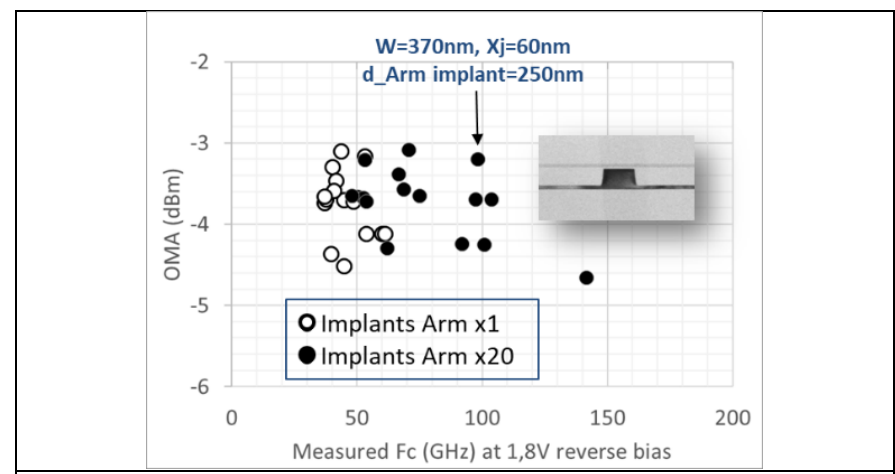

Fig 6: Confirmation of best geometry confirmed with cut-off frequencies measured at $1,8 \mathrm{~V}$ reverse bias on dedicated $\mathrm{RF}$ structures. $\mathrm{L}=2,4 \mathrm{~mm}$.

Then we optimized the value of the Waveguide (Wells) implants by increasing sequentially the dose of Pwell and Nwell vs the reference. The figure 7 show that we improved OMA by increasing both values by a 1,5 factor.
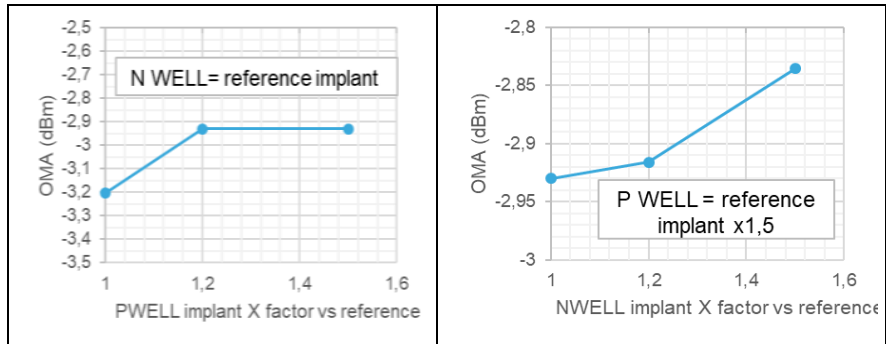

Fig.7: (left) Impact of Si guide Pwell implant increase for a given Nwell value and (right) Impact of $\mathrm{Si}$ guide Nwell implant increase for the best Nwell implant. OMA is optimum for the highest values. $\mathrm{L}=2,4 \mathrm{~mm}$.

Finally, compared to our previous industrial platform (Rib modulators in [1]) we can see on figure 8 that the optimized Deep Rib structure improves the OMA by $1,6 \mathrm{~dB}$ with an improved cut off frequency (1/2PiRC).

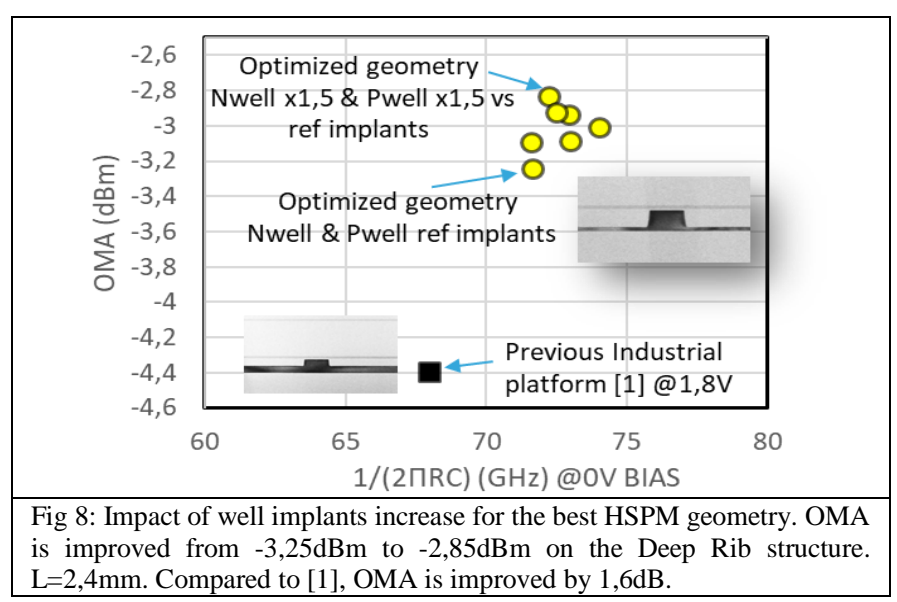


The figure 9 provides OMA vs L characteristic for the best device.

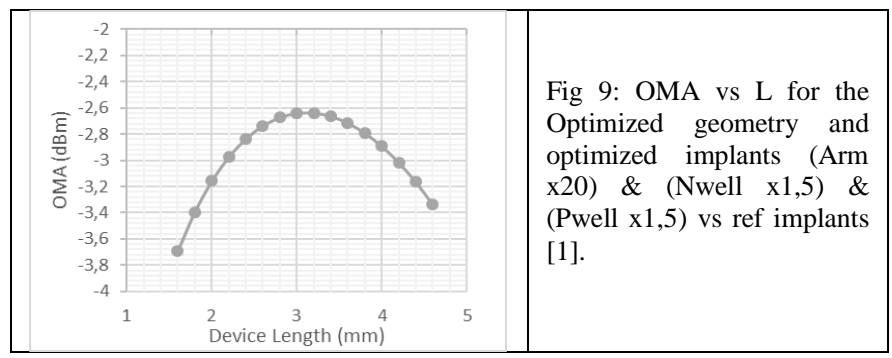

IV. NEW DEEP RIB DEVICES INTEGRATION WITH Z IMPLANTS

We propose in this section the combination of our best DeepRib geometry with Z-implants (combination of depths of vertical $\mathrm{N}$-well implants ) to improve the coverage between the highly confined mode in the Deep-Rib structure and the depletion zone of the PN junction (fig.10).

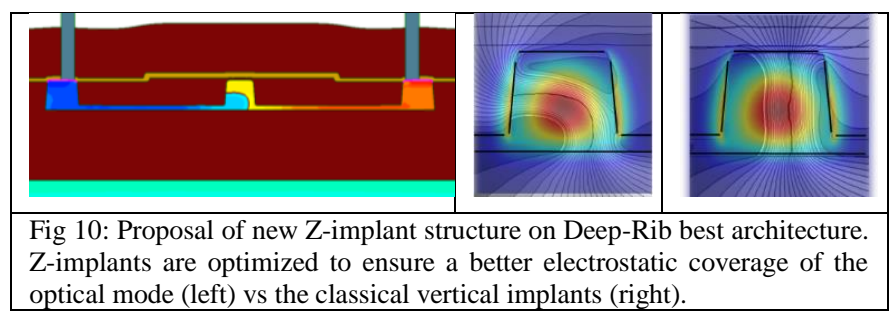

The same DOE was analyzed with these Z-implants and the best geometry was the same as previously identified, but with $\mathrm{Xj}=140 \mathrm{~nm}$ to ensure the best overlap with the junction. Fig.11 \& 12 shows the optical performances of the structure and compare it with the previous points. One can see that this approach improves OMA by $2,15 \mathrm{~dB}$ compared to our previous platform, by assuming a slight decrease of the cut-off frequency.

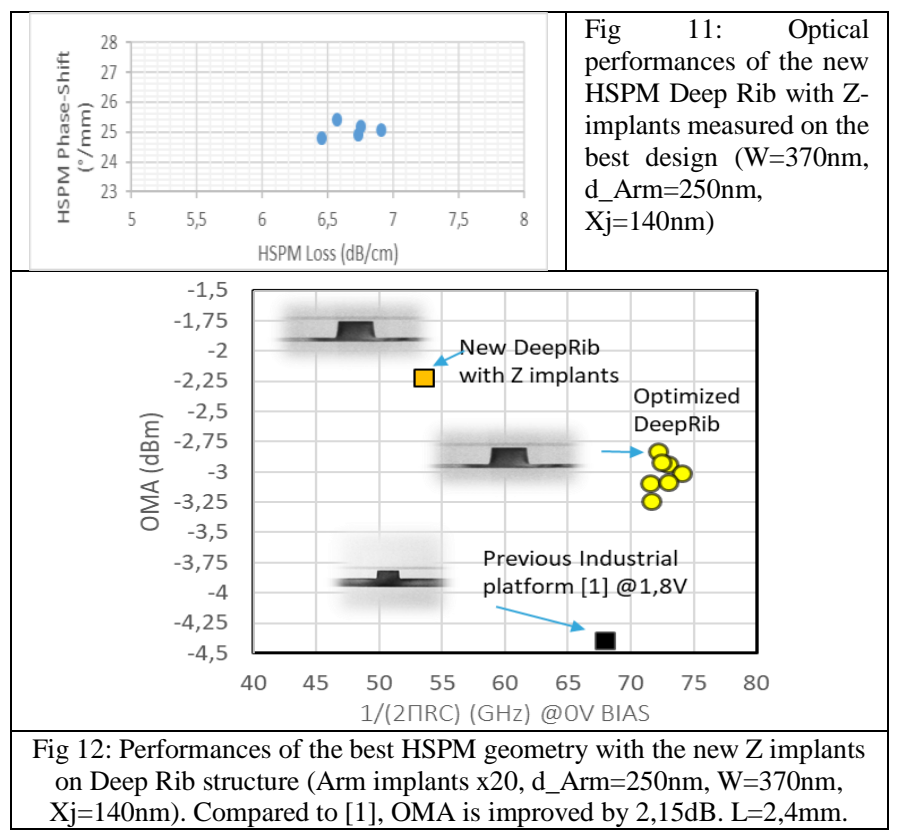

The figure 13 provides OMA vs L characteristic for the best device.

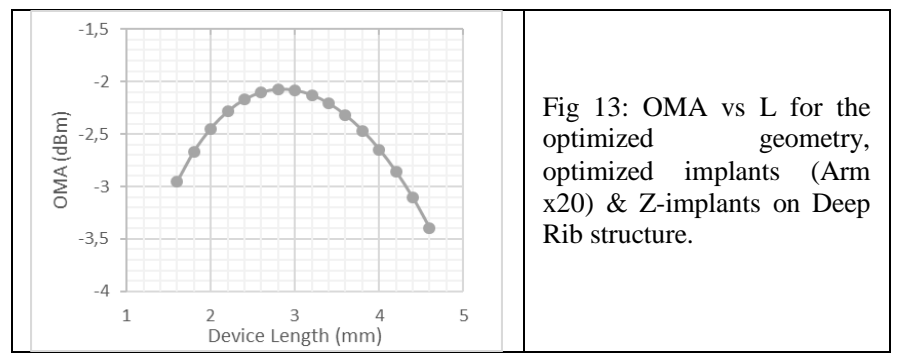

Finally, we compared the 3 structures: Rib modulator from [1], the optimized vertical PN Deep-Rib modulator (from fig.8) and the Deep-Rib modulator with Z-implants. OMA (calculated based on experimental data) in function of device length graphs and $\mathrm{V}_{\mathrm{Pi}_{\mathrm{i}}} *_{\mathrm{Pi}}$ highlight the improvement proposed by those structures compared to the reference platform.

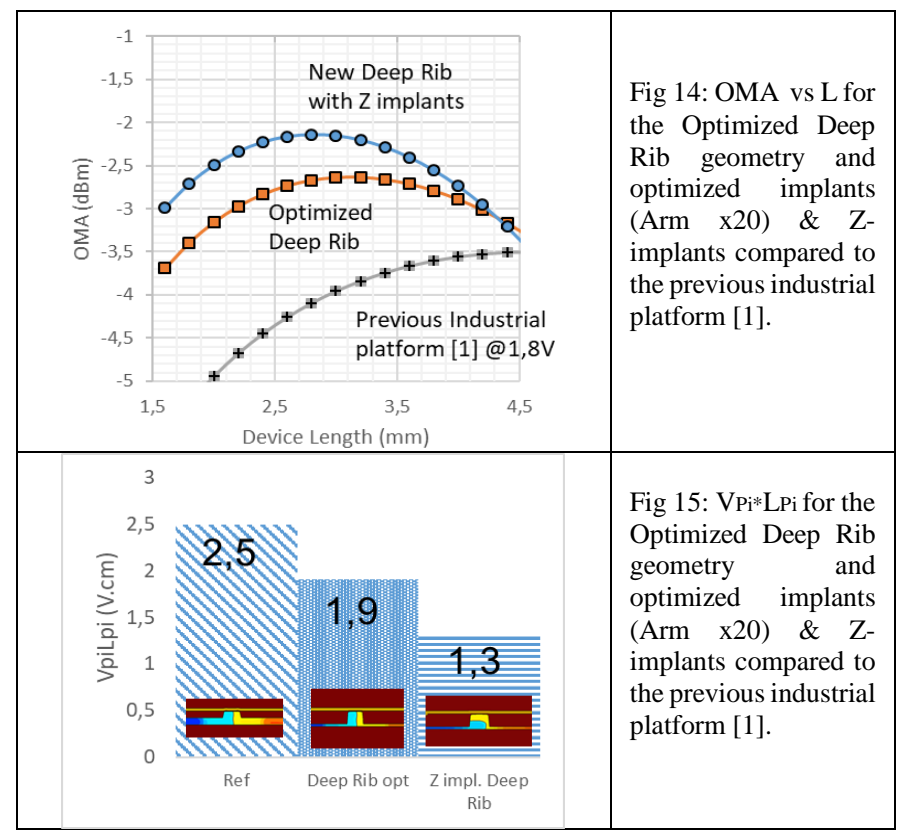

\section{CONCLUSION}

This paper shows optimized deep Rib modulators for 400G and above applications. DOE integration allowed us to define the optimal structure and implants to guaranty high speed (high cutoff frequency) coupled with high optical performances, to propose highly performant silicon $\mathrm{PN}$ junction modulator with OMA improved by $1,6 \mathrm{~dB}$ and up to $2,15 \mathrm{~dB}$ compared to the industrial state-of-the-art. The best Deep Rib geometry with Zimplants has OMA (Optical Modulation Amplitude) value as high as $-2 \mathrm{dBm}(\mathrm{L}=3 \mathrm{~mm})$ with $54 \mathrm{GHz} @ 0 \mathrm{~V}$ cut-off frequency.

This work was partially supported by $\mathrm{H} 2020$ project $\mathrm{N}^{\circ} 688516$ COSMICC and project IRT Nanoelec.

[1] F.Boeuf et al., "Silicon Photonics $R \& D$ and Manufacturing on 300-mm Wafer Platform" JOURNAL OF LIGHTWAVE TECHNOLOGY, VOL. 34, NO. 2, JANUARY 15, 2016

[2] E. Temporiti et al. Solid State Elec. 2016, 